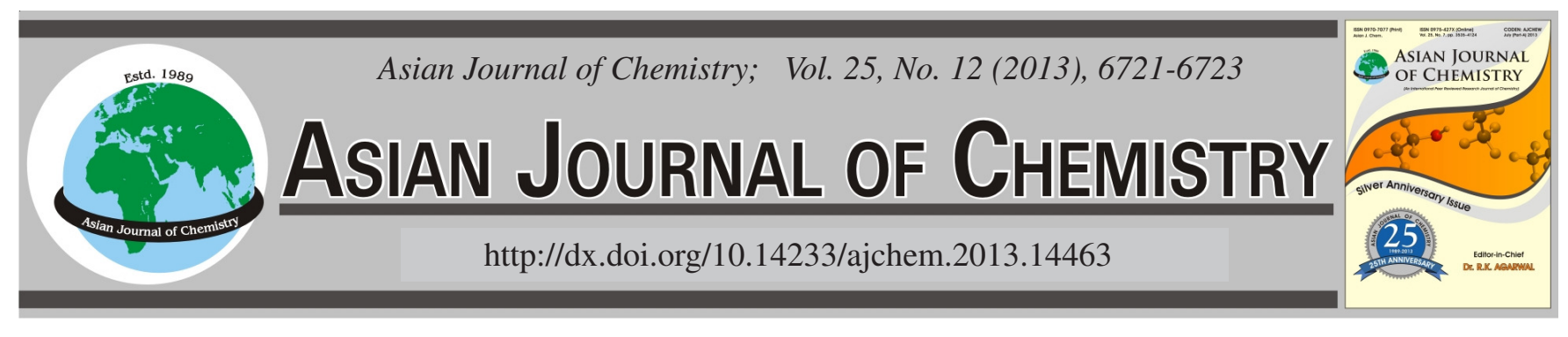

\title{
Synthesis of Chiral Geminal Dicationic Ionic Liquid from Amino Acids
}

\author{
Aiwu Yin ${ }^{1}$, Saijin Huang ${ }^{2, *}$ and ChuAnBin Wu ${ }^{2}$
}

\begin{abstract}
${ }^{1}$ Department of Life Science and Chemistry, Hunan University of Science and Engineering, Yongzhou, P.R. China ${ }^{2}$ Department of Chemistry Engineering, Hunan Institute of Engineering, Xiangtan, P.R. China

*Corresponding author: E-mail: huangsaijin@sina.cn
\end{abstract}

Chiral imidazolium ionic liquids (3) from amino acid were synthesized when reacted with 1,2-dibromoethane, 1,3-dibromopropane, 1,4dibromobutane, 1,6-dibromohexane and 1,9-dibromononane, respectively, to obtain a series of chiral geminal dicationic ionic liquid (5), The structures of the synthesized compouds were determined by IR, ${ }^{1} \mathrm{H}$ and ${ }^{13} \mathrm{C} \mathrm{NMR}$, this method for the synthesis of chiral geminal dicationic ionic liquid is simple and rapid with high yield and the study for their application is under way.

Key Words: Chiral imidazolium ionic liquids, Chiral geminal dicationic ionic liquid, Synthesize, Characterization.

\section{INTRODUCTION}

Since the first report of geminal dicationic ionic liquids in $2003^{1}$, much effort has been devoted to the geminal dicationic ionic liquids because their superior physical properties compared to traditional monocationic ionic liquids ${ }^{2}$. The application as solvents in high-temperature reactions ${ }^{3}$, novel high-temperature lubricants ${ }^{4,5}$, ultrastable separation phases ${ }^{6}$ and dye sensitized solar cells ${ }^{7,8}$ have been reported.

It is well known that the chiral monocationic ionic liquids are attractive due to their potential for asymmetric synthesis ${ }^{9,10}$, gas chromatography ${ }^{11}$, optical resolution of racemates ${ }^{12}$, stereo selective polymerization ${ }^{13}$. But there is no report on the synthesis and application of chiral geminal dicationic ionic liquids.

In this work, chiral imidazolium ionic liquids were designed from the natural amino acid, then reacted with 1,2dibromoethane, 1,3-dibromopropane, 1,4-dibromobutane, 1,6dibromohexane and1,9-dibromononane, respectively, to obtain a series of chiral geminal dicationic ionic liquid (Scheme-I), the structures of the synthesized compouds were determined by IR, ${ }^{1} \mathrm{H}$ NMR, ${ }^{13} \mathrm{C}$ NMR and the study for their application research in synthesis and separation is under way.

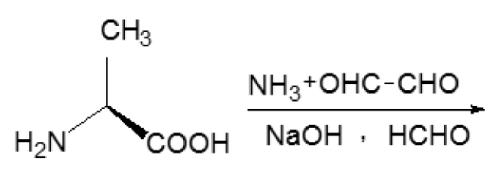

1

a $\mathrm{R}=-\mathrm{CH}_{3}$<smiles>[R]C(C(=O)O[Na])n1cncc1C1CC1</smiles>

2<smiles>[R]C(OCC)n1ccnc1</smiles>

3<smiles>[R]C(C(=O)OCC)n1cc[n+](C(C)n2cc(Br)cc2Br)c1</smiles>

4<smiles>[R]COC(=O)OCC</smiles><smiles>ClC1CC1</smiles>

$\mathrm{n}=2,3,4,6,9$<smiles>[R]C(C(=O)O)n1cc[n+](C(C)n2cc[n+]([B-])c2)c1</smiles>

5

\footnotetext{
Scheme-I: Synthetic route of chiral geminal dicationic ionic liquid
} 


\section{EXPERIMENTAL}

All the chemical reagents used are of analytical pure grade,1,2-dibromoethane, 1,3-dibromopropane, 1,4-dibromobutane, 1,6-dibromohexane and 1,9-dibromononane was distilled again. Chiral imidazolium ionic liquids $\mathbf{3}$ from amino acid were synthesized according to the literature procedures ${ }^{14}$ and the products were determined by IR, ${ }^{1} \mathrm{H}$ NMR.

Melting points were recorded on a digital microscope are uncorrected. IR $(\mathrm{KBr})$ spectra $\left(\mathrm{V} \mathrm{cm}^{-1}\right)$ were obtained on Nicolet AVATAR 370 spectrometer, ${ }^{1} \mathrm{H}$ and ${ }^{13} \mathrm{C}$ NMR spectra were taken on a Avance 400 NMR spectrometer, using TMS as internal standard. The specific rotations were measured on an Optical Instrument Ltd. WZZ-ZS polarimeter made in Shanghai Jinke.

Procedure for the synthesis of chiral geminal dicationic ionic liquid (5): 0.25 molar of 1,2-dibromoethane, 1,3-dibromopropane, 1,4-dibromobutane, 1,6-dibromohexane and 1,9dibromononane, resprctively, reacted with 0.5 molar 3 under the protection of $\mathrm{N}_{2}$ at room temperature then all the products were purified by extraction by $200 \mathrm{~mL}$ ethyl acetate four times when reaction solution changed into viscous and vacuum drying $72 \mathrm{~h}$ to get product $\mathbf{4}$, which on refluxing in $20 \mathrm{~mL}$ concentrated hydrochloric acid for $4 \mathrm{~h}$, evaporated solvent under reduced pressure to obtain crude products and then recrystallized to afford the products 5 .

\section{RESULTS AND DISCUSSION}

Melting points and optical activity of chiral geminal dicationic ionic liquid: Generally speaking, most ionic liquid are low melting, called room temperature ionic liquids, but the synthesized chiral geminal dicationic ionic liquid $\mathbf{5}$ have high melting (Table-1) and with the linkage chains increase, the melting pionts decrease.

TABLE-1

MELTING POINTS AND OPTICAL ACTIVITY OF 5

\begin{tabular}{ccc}
\hline Compound & $\mathrm{T}\left({ }^{\circ} \mathrm{C}\right)$ & {$[\alpha]_{\mathrm{D}}{ }^{25}$} \\
\hline $\mathbf{5 a}_{\mathbf{2}}$ & 218 & $+7.4\left(\mathrm{C} 2.0 \%, \mathrm{CH}_{3} \mathrm{OH}\right)$ \\
$\mathbf{5 a}_{\mathbf{3}}$ & 211 & $+7.8\left(\mathrm{C} 2.0 \%, \mathrm{CH}_{3} \mathrm{OH}\right)$ \\
$\mathbf{5 a}_{\mathbf{4}}$ & 205 & $+8.3\left(\mathrm{C} 2.0 \%, \mathrm{CH}_{3} \mathrm{OH}\right)$ \\
$\mathbf{5 a}_{\mathbf{6}}$ & 197 & $+9.4\left(\mathrm{C} 2.0 \%, \mathrm{CH}_{3} \mathrm{OH}\right)$ \\
$\mathbf{5 a}_{9}$ & 164 & $+10.6\left(\mathrm{C} 2.0 \%, \mathrm{CH}_{3} \mathrm{OH}\right)$ \\
$\mathbf{5 b}_{\mathbf{2}}$ & 224 & $-18.4\left(\mathrm{C} 2.0 \%, \mathrm{CH}_{3} \mathrm{OH}\right)$ \\
$\mathbf{5 b}_{\mathbf{3}}$ & 216 & $-18.9\left(\mathrm{C} 2.0 \%, \mathrm{CH}_{3} \mathrm{OH}\right)$ \\
$\mathbf{5 b}_{\mathbf{4}}$ & 208 & $-19.4\left(\mathrm{C} 2.0 \%, \mathrm{CH}_{3} \mathrm{OH}\right)$ \\
$\mathbf{5 b}_{\mathbf{6}}$ & 201 & $-20.3\left(\mathrm{C} 2.0 \%, \mathrm{CH}_{3} \mathrm{OH}\right)$ \\
$\mathbf{5 b}_{\mathbf{9}}$ & 171 & $-22.8\left(\mathrm{C} 2.0 \%, \mathrm{CH}_{3} \mathrm{OH}\right)$ \\
\hline
\end{tabular}

${ }^{a}$ Melting points were recorded on a digital microscope and are uncorrected.

Synthesis of chiral geminal dicationic ionic liquid: The synthesis for chiral geminal dicationic ionic liquid $\mathbf{5}$ is easy to purify and have high yields and the products were determined by IR, ${ }^{1} \mathrm{H}$ NMR (Table-2), ${ }^{13} \mathrm{C}$ NMR (Table-3). Compounds 5 in IR showed absroption at $3424 \mathrm{~cm}^{-1}(-\mathrm{OH}), 1736(\mathrm{C}=\mathrm{O})$, 1629, 1579 ((imidazole).

Solubility of chiral geminal dicationic ionic liquid: Great attention has been paid to the ionic liquid as a new type of green solvent. The solubility of the synthesized chiral

\begin{tabular}{|c|c|}
\hline & $\begin{array}{c}\text { TABLE-2 } \\
{ }^{1} \mathrm{H} \text { NMR SPECTRA OF COMPOUNDS } 5\end{array}$ \\
\hline Compound & ${ }^{1} \mathrm{H}$ NMR, $\delta(\mathrm{ppm})$ \\
\hline $5 \mathbf{a}_{2}$ & $\begin{array}{l}8.76(\mathrm{~s}, 2 \mathrm{H}), 7.40(\mathrm{~s}, 2 \mathrm{H}), 7.34(\mathrm{~s}, 2 \mathrm{H}), 4.82(\mathrm{q}, 2 \mathrm{H}), 3.72 \\
(\mathrm{t}, 4 \mathrm{H}), 1.60(\mathrm{~d}, 6 \mathrm{H}) .\end{array}$ \\
\hline $5 \mathbf{a}_{3}$ & $\begin{array}{l}8.77(\mathrm{~s}, 2 \mathrm{H}), 7.43(\mathrm{~s}, 2 \mathrm{H}), 7.34(\mathrm{~s}, 2 \mathrm{H}), 4.80,(\mathrm{q}, 2 \mathrm{H}), 3.80 \\
(\mathrm{t}, 4 \mathrm{H}), 1.71(\mathrm{~m}, 2 \mathrm{H}), 1.62(\mathrm{~d}, 6 \mathrm{H}) .\end{array}$ \\
\hline $5 \mathbf{a}_{4}$ & $\begin{array}{l}8.84(\mathrm{~s}, 2 \mathrm{H}), 7.46(\mathrm{~s}, 2 \mathrm{H}), 7.41(\mathrm{~s}, 2 \mathrm{H}), 4.78(\mathrm{~d}, 2 \mathrm{H}), 3.76 \\
(\mathrm{t}, 4 \mathrm{H}), 1.75(\mathrm{~m}, 4 \mathrm{H}), 1.61(\mathrm{~d}, 6 \mathrm{H}) .\end{array}$ \\
\hline $5 \mathbf{a}_{6}$ & $\begin{array}{l}8.89(\mathrm{~s}, 2 \mathrm{H}), 7.48(\mathrm{~s}, 2 \mathrm{H}), 7.37(\mathrm{~s}, 2 \mathrm{H}), 4.87(\mathrm{q}, 2 \mathrm{H}), 3.78 \\
(\mathrm{t}, 4 \mathrm{H}), 1.86(\mathrm{~m}, 4 \mathrm{H}), 1.56(\mathrm{~d}, 6 \mathrm{H}), 1.30(\mathrm{~m}, 4 \mathrm{H}) .\end{array}$ \\
\hline $5 \mathbf{a}_{9}$ & $\begin{array}{l}8.85(\mathrm{~s}, 2 \mathrm{H}), 7.44(\mathrm{~s}, 2 \mathrm{H}), 7.35(\mathrm{~s}, 2 \mathrm{H}), 4.88(\mathrm{q}, 2 \mathrm{H}), 3.79 \\
(\mathrm{t}, 4 \mathrm{H}), 1.88(\mathrm{~m}, 4 \mathrm{H}), 1.58(\mathrm{~d}, 6 \mathrm{H}), 1.33-1.40(\mathrm{~m}, 10 \mathrm{H}) .\end{array}$ \\
\hline $5 \mathbf{b}_{2}$ & $\begin{array}{l}8.76(\mathrm{~s}, 2 \mathrm{H}), 7.40(\mathrm{~s}, 2 \mathrm{H}), 7.36(\mathrm{~s}, 2 \mathrm{H}), 4.82(\mathrm{q}, 2 \mathrm{H}), 3.72 \\
(\mathrm{t}, 4 \mathrm{H}), 2.22(\mathrm{~m}, 2 \mathrm{H}), 1.01(\mathrm{~d}, 6 \mathrm{H}), 0.80(\mathrm{~d}, 6 \mathrm{H}) .\end{array}$ \\
\hline $5 \mathbf{b}_{3}$ & $\begin{array}{l}8.76(\mathrm{~s}, 2 \mathrm{H}), 7.42(\mathrm{~s}, 2 \mathrm{H}), 7.38(\mathrm{~s}, 2 \mathrm{H}), 4.81,(\mathrm{q}, 2 \mathrm{H}), 3.79 \\
(\mathrm{t}, 4 \mathrm{H}), 2.22(\mathrm{~m}, 2 \mathrm{H}), 1.73(\mathrm{~m}, 2 \mathrm{H}), 1.02(\mathrm{~d}, 6 \mathrm{H}), 0.84(\mathrm{~d}, \\
6 \mathrm{H}) .\end{array}$ \\
\hline $5 b_{4}$ & $\begin{array}{l}8.87(\mathrm{~s}, 2 \mathrm{H}), 7.48(\mathrm{~s}, 2 \mathrm{H}), 7.42(\mathrm{~s}, 2 \mathrm{H}), 4.80(\mathrm{~d}, 2 \mathrm{H}), 3.78 \\
(\mathrm{t}, 4 \mathrm{H}), 2.22(\mathrm{~m}, 2 \mathrm{H}), 1.76(\mathrm{~m}, 4 \mathrm{H}), 1.04(\mathrm{~d}, 6 \mathrm{H}), 0.87(\mathrm{~d}, \\
6 \mathrm{H}) .\end{array}$ \\
\hline $5 \mathbf{b}_{6}$ & $\begin{array}{l}\text { 9.01(s, 2H), 7.68(s, 2H), 7.67(s, 2H), } 4.80(\mathrm{br}, 2 \mathrm{H}), 3.73 \\
(\mathrm{~m}, 4 \mathrm{H}), 2.21(\mathrm{~m}, 2 \mathrm{H}), 1.87(\mathrm{~m}, 4 \mathrm{H}), 1.32(\mathrm{~m}, 4 \mathrm{H}), 1.06 \\
(\mathrm{~d}, 6 \mathrm{H}), 0.80(\mathrm{~d}, 6 \mathrm{H}) .\end{array}$ \\
\hline $5 \mathbf{b}_{9}$ & $\begin{array}{l}8.89(\mathrm{~s}, 2 \mathrm{H}), 7.62(\mathrm{~s}, 2 \mathrm{H}), 7.58(\mathrm{~s}, 2 \mathrm{H}), 4.80(\mathrm{q}, 2 \mathrm{H}), \\
3.81(\mathrm{t}, 4 \mathrm{H}), 1.86(\mathrm{~m}, 4 \mathrm{H}), 2.24(\mathrm{~m}, 2 \mathrm{H}), 1.32-1.39(\mathrm{~m}, \\
10 \mathrm{H}), 1.01(\mathrm{~d}, 6 \mathrm{H}), 0.85(\mathrm{~d}, 6 \mathrm{H}) .\end{array}$ \\
\hline $\begin{array}{l}{ }^{1} \mathrm{H} \text { NMR } \\
\text { standard. }\end{array}$ & ectra were taken in $\mathrm{D}_{2} \mathrm{O}(\mathbf{5})$, using TMS as internal \\
\hline & $\begin{array}{c}\text { TABLE-3 } \\
{ }^{13} \mathrm{C} \text { NMR SPECTRA OF COMPOUNDS } 5 \\
\end{array}$ \\
\hline Compound & ${ }^{13} \mathrm{C} \mathrm{NMR}, \delta(\mathrm{ppm})$ \\
\hline $5 \mathbf{a}_{2}$ & $14.6,25.4,58.6,122.4,123.6,138.7,172.2$ \\
\hline $5 \mathbf{a}_{3}$ & $14.8,25.2,34.8,58.9,122.3,123.4,137.4,172.2$ \\
\hline $5 \mathbf{a}_{4}$ & $14.8,25.6,38.6,64.6,122.8,123.0,137.4,172.2$ \\
\hline $5 \mathbf{a}_{6}$ & $15.0,27.3,30.3,54.8,72.4,122.8,123.6,137.3,172.2$ \\
\hline $5 \mathbf{a}_{9}$ & $\begin{array}{l}\text { 15.1, 26.3, 28.3, 30.4, 32.5, 54.8, 72.2, 122.7, 123.6, } \\
137.2,172.1\end{array}$ \\
\hline $5 \mathbf{b}_{2}$ & $17.3,25.3,28.9 .53 .1,70.7,122.4,123.5,138.7,172.2$ \\
\hline $5 \mathbf{b}_{3}$ & $17.4,25.2,28.3,52.3,71.7,122.3,123.4,137.4,172.2$ \\
\hline $5 \mathbf{b}_{4}$ & $17.9,25.3,30.4,52.1,72.5,122.5,123.7,137.4,172.1$ \\
\hline $5 \mathbf{b}_{6}$ & $\begin{array}{l}18.2,25.2,27.6,30.5,55.0,73.2,120.3,123.4,137.0 \text {, } \\
172.2\end{array}$ \\
\hline $5 \mathbf{b}_{9}$ & 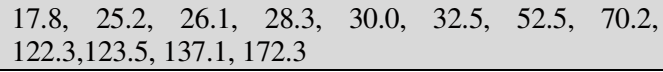 \\
\hline
\end{tabular}

geminal dicationic ionic liquid in different solvent were investigated (Table-4). It was found that all the chiral geminal dicationic ionic liquid synthesized were dissolved in water and methanol and insoluble in ethyl acetate, acetone, chloroform, which is quite similar to the monocationic ionic liquids with $\mathrm{Br}^{-}$as anion.

\section{Conclusion}

A synthetic route toward a novel chiral geminal dicationic ionic liquid from amino acids has been described. The solubility and other physical properties have been preliminary studied, further investigations for their application is under way.

\section{ACKNOWLEDGEMENTS}

This work is supported by the Construct Program of the Key Discipline in Hunan Province (2012) and Aid Program for Science and Technology Innovative Research Team in Higher Educational Institutions of Hunan Province (2012-318) and 


\begin{tabular}{|c|c|c|c|c|c|}
\hline \multicolumn{6}{|c|}{$\begin{array}{c}\text { TABLE-4 } \\
\text { SOLUBILITIES OF THE CHIRAL GEMINAL } \\
\text { DICATIONIC IONIC LIQUID }\end{array}$} \\
\hline \multirow{2}{*}{$\begin{array}{c}\text { Chiral } \\
\text { geminal } \\
\text { dicationic } \\
\text { ionic liquid }\end{array}$} & \multicolumn{5}{|c|}{ Solubility } \\
\hline & Water & Methanol & $\begin{array}{l}\text { Ethyl } \\
\text { acetate }\end{array}$ & Acetone & Chloroform \\
\hline $5 \mathbf{a}_{2}$ & $\mathrm{~s}$ & $\mathrm{~s}$ & ins & ins & ins \\
\hline $5 \mathbf{a}_{3}$ & s & s & ins & ins & ins \\
\hline $5 \mathbf{a}_{4}$ & $\mathrm{~s}$ & $\mathrm{~s}$ & ins & ins & ins \\
\hline $5 \mathbf{a}_{6}$ & s & $\mathrm{s}$ & ins & ins & ins \\
\hline $5 \mathbf{a}_{9}$ & s & s & ins & ins & ins \\
\hline $5 b_{2}$ & s & $\mathrm{s}$ & ins & ins & ins \\
\hline $5 \mathbf{b}_{3}$ & s & $\mathrm{s}$ & ins & ins & ins \\
\hline $5 \mathbf{b}_{4}$ & s & $\mathrm{s}$ & ins & ins & ins \\
\hline $5 b_{6}$ & s & $\mathrm{s}$ & ins & ins & ins \\
\hline $5 b_{9}$ & $\mathrm{~s}$ & $\mathrm{~s}$ & ins & ins & ins \\
\hline
\end{tabular}

Science and Technology Planning Project of Hunan Province (no. 2013FJ3004) and the Key Scientific Research Project of the Hunan Provincial Education Department (Grant No. 2013A).

\section{REFERENCES}

1. R.P. Singh and J.M. Shreeve, J. Chem. Soc., Chem. Commun., 1366 (2003).

2. J.L. Anderson, R. Ding, A. Ellern and D.W. Armstrong, J. Am. Chem. Soc., 127, 593 (2005).

3. X.X. Han and D.W. Armstrong, Org. Lett., 7, 4205 (2005).

4. C.M. Jin, C.F. Ye, B.S. Phillips, J.S. Zabinski, J.M. Shreeve, X.Q. Liu, W.M. Liu and J.M. Shreeve, J. Mater. Chem., 16, 1529 (2006).

5. Z. Zhuo, B.S. Phillips, J.-C. Xiao and J.M. Shreeve, Chem. Mater, 20, 2719 (2008).

6. Q.B. Liu, F.V. Rantwijk and R.A. Sheldon, J. Chem. Technol. Biotechnol, 81, 401 (2006).

7. C. Zafer, K. Ocakoglu, C. Ozsoy and S. Icli, Electrochim. Acta, 54, 5709 (2009).

8. J.Y. Kim, T.H. Kim and D.Y. Kim, N.-G. Park and K.-D. Ahn, J. Power Sources, 175, 692 (2008).

9. Z.M. Wang, Q. Wang, Y. Zhang and W.L. Bao, Tetrahedron Lett., 46, 4657 (2005).

10. B. Pegot, G. Vo-Thanh, D. Gori and A. Loupy, Tetrahedron Lett., 45, 6425 (2004).

11. J. Ding, T. Welton and D.W. Armstrong, Anal. Chem., 76, 6819 (2004).

12. J. Ding, V. Desikan, X.X. Han, T.L. Xiao, R.F. Ding, W.S. Jenks and D.W. Armstrong, Org. Lett., 7, 335 (2005).

13. C.J. Adams, M.J. Earle and K.R. Seddon, Chem. Commun., 1043 (1999).

14. W.L. Bao, Z.M. Wang and Y.X. Li, J. Org. Chem., 68, 591 (2003). 